\title{
Short Review of Ischemia- and Hypoxia-Protective Roles of “Big Potassium" (BK) Channels
}

\author{
John F. Peppin ${ }^{1,2}$, Joseph V. Pergolizzi33,4,5, Alexander Kraus ${ }^{3}$, Robert B. Raffa ${ }^{3,5,6,7}$
}

${ }^{1}$ Marian University College of Osteopathic Medicine, Indianapolis, USA

${ }^{2}$ Pikeville University College of Osteopathic Medicine, Pikeville Kentucky, USA

${ }^{3}$ Enalare Therapeutics Inc., Princeton, USA

${ }^{4}$ NEMA Research Inc., Naples, USA

${ }^{5}$ Neumentum Inc., Morristown, USA

${ }^{6}$ University of Arizona College of Pharmacy, Tucson, USA

${ }^{7}$ Temple University School of Pharmacy, Philadelphia, USA

Email: ^robert.raffa@gmail.com

How to cite this paper: Peppin, J.F., Pergolizzi, J.V., Kraus, A. and Raffa, R.B. (2021) Short Review of Ischemia- and Hypoxia-Protective Roles of "Big Potassium" (BK) Channels. Journal of Biosciences and Medicines, 9, 150-160.

https://doi.org/10.4236/jbm.2021.96014

Received: May 19, 2021

Accepted: June 20, 2021

Published: June 23, 2021

Copyright ( 2021 by author(s) and Scientific Research Publishing Inc. This work is licensed under the Creative Commons Attribution International License (CC BY 4.0).

http://creativecommons.org/licenses/by/4.0/

(c) (i) Open Access

\begin{abstract}
There is accumulating evidence that the subfamily of large-conductance potassium ("big", "BK") channels are involved in diverse, and perhaps coordinated, protective or counteractive responses to local or generalized ischemia and hypoxia. Although widely distributed, the physiological differences among BK channels which results from posttranslational modification (alternative splicing) and co-assembly with auxiliary modulatory subunits $\left(\beta_{1-4}\right.$ and $\left.\gamma_{1-4}\right)$, bestows localized differences in subunit composition, distribution, $2^{\text {nd }}$-messenger coupling, and pharmacologic properties. Due to the ubiquitous nature of BK channels and the multiplicity of subtypes, they have many potential therapeutic applications in the maintenance of oxygen homeostasis, cerebro- and cardio-protection, and stimulation of respiration in response to drug-induced respiratory depression. BK channels may also offer other potentially broad and underrecognized promising targets for novel pharmaceutical development.
\end{abstract}

\section{Keywords}

Big potassium Channels, $\mathrm{BK}_{\mathrm{Ca}}$, Ischemia, Hypoxia, Respiratory Stimulation, ENA-001

\section{Introduction}

G-protein coupled receptors (GPCRs) are ubiquitous transmembrane receptor 
proteins that are major targets in disease-treatment and drug-development [1]. They are currently the most common targets for FDA (United States Food and Drug Administration)-approved drugs [2]. Although the GPCRs are more commonly focused upon, BK (big potassium) channels are perhaps even more fundamental. Due to variability in component composition and posttranslational modifications, BK channel subtypes are expressed in different frequencies in various organs/tissues, with differing chemo-sensitivities and $2^{\text {nd }}$-messenger transduction processes. They are involved in various neurological processes and their dysfunction for example leads to neurological pathologies like epilepsy, paroxysmal dyskinesia, or schizophrenia. In places where their signaling is involved in the regulation of blood flow and the detection of deviations from normal $\mathrm{pO}_{2}, \mathrm{pCO}_{2}$, and $\mathrm{pH}$, the macroscopic physiologic responses result in protection against episodes of ischemia and hypoxia. This includes cardiac and cerebral ischemia and hypoxia, and the stimulation of respiration. We present a short review of these topics.

\section{BK Channels}

There are three main subfamilies of $\mathrm{Ca}^{2+}$-activated potassium channels, SK (small conductance), IK (intermediate conductance), and BK (big conductance) [3]. The BK channels (encoded by the Kcnmal gene) are also called $\mathrm{BK}_{\mathrm{Ca}}$, MaxiK, Slo1, KCa1.1, and KCNMA1, among other names [4]. They were first cloned in 1992, and based on their modulatory roles have been described as the "universal regulator of cellular excitability" and even "king of ion channels" [5] [6]. $\mathrm{BK}$ channels are voltage- and $\mathrm{Ca}^{2+}$-sensitive potassium channels, formed as tetramers of $\alpha$ subunits [6]. The channel is formed by the interface of the four subunits [7]. BK $\alpha$ subunits differ from the SK/IK group in the existence of an additional transmembrane helix which drives the $N$-terminus to the extracellular side of the plasma membrane. Each membrane-spanning domain of a BK channel contains a pore-gate and voltage-sensing domain [8]. These two domains are composed of two regulators of conductance of potassium, RCK1 and RCK2 [9]. The RCK1-RCK2 link with BK channels is highly conserved (Figure 1) [10].

An important aspect of BK channel function that they allow rapid and large influxes of potassium through the channel, thus hyperpolarizing the membrane. Conformational change of the subunits and channels transduces and stabilizes the channel pore in its open state [11]. The large ("big") influx is 10 - 20 times larger than most other $\mathrm{K}^{+}$channels [6]. They can form homo- and hetero-multimeric channels and can be expressed on both pre and post synaptic sites [12] [13] [14]. BK channels can detect intracellular calcium concentrations as well as membrane depolarization independently. This puts the BK channel in a "unique" position to sense (monitor) the state of activity of excitable cells [15]. And the expression of particular BK subunit subtypes tune the BK channels to the local signaling environment (unless otherwise specified, we will use the term to mean BK channels in general) [14]. The different subunit subtypes differentiate the 


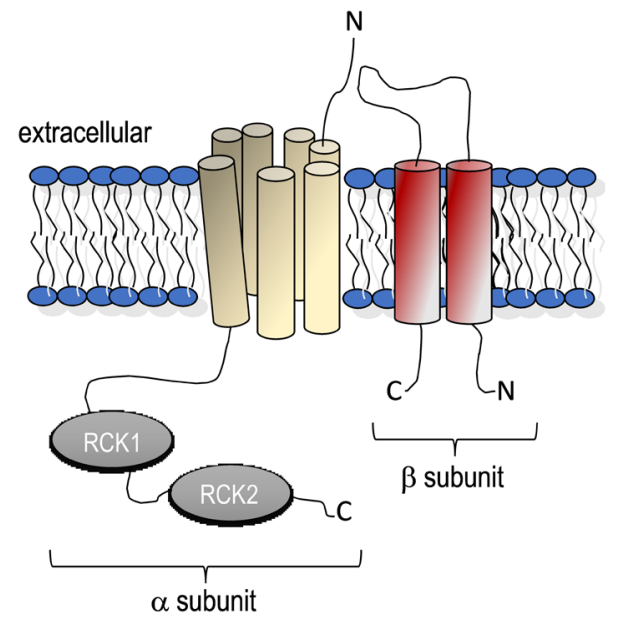

Figure 1. Representation of a transmembrane BK channel, showing one of the tetrameric $\alpha$ subunits with $\mathrm{Ca}^{2+}$-sensing RCK1 and RCK2, and accessory $\beta$ subunit. The $\mathrm{K}^{+}$pore ("channel") is formed at the interface of the four $\alpha$ subunits. Variations in each of the components provides diversity in sensitivity and response.

functional expression of these channels. BK channel currents abruptly increase during the first two weeks after birth in animal models [16], which may be coincident with their functional maturation as the channels adjust (to) neuronal properties as the animal matures. This is an "experience dependent plasticity" that helps shape how BK channels will function in the mature animal [15] [17].

For most ion channels, functional identity is defined by properties conferred by the pore-forming subunits of the channel [14]. In contrast, an enormous diversity in BK channel function arises from a merging together with non-poreforming regulatory subunits. In fact, the same BK pore-forming subunits can participate in channels that could be considered to be entirely functionally distinct, as a simple consequence of the "wardrobe" of (associated) regulatory subunits that can decorate the pore-forming subunits [14].

\section{Cerebral Ischemia}

During ischemia-induced reduction in blood flow, critical supplies of oxygen and glucose to cells are impeded or stopped. These results in a decoupling of oxidative phosphorylation, reduced ATP levels, disruption of ionic flow, and disruption of normal ionic gradients. This leads to, for example, depolarization of cerebral cellular membranes, with resultant rapid influx of $\mathrm{Ca}^{2+}$ and release of the excitatory amino acid, glutamate [15]. Normally, glutamate is safely cleared from the synaptic cleft, but the processes are overwhelmed during ischemia. The excess glutamate in synaptic clefts binds to NMDA ( $N$-methyl-D-aspartate) and AMPA ( $\alpha$-amino-3-hydroxy-5-methyl-4-isoxazolepropionic acid) receptors, producing a wave of depolarization that further propagates the ischemic damage [18].

BK channels function as an "emergency brake" by limiting calcium-enhanced glutamate release and NMDA activity that occurs during cerebral ischemia [15]. 
For example, in a model of permanent occlusion of the middle cerebral artery in spontaneously hypertensive rats, intravenous administration of the fluorooxindole BK channel opener BMS-204352 ((3S)-(+)-(5-Chloro-2-methoxyphenyl)-1,3-dihydro-3-fluoro-6-(trifluoromethyl)-2H-indole-2-one) reduced cortical infarct volume [19]. But a phase-3 study with BMS-204352 in humans in the setting of acute ischemic stroke was not positive [15] [19]. The reasons for this failure are not clear [20].

Blood flow in brain is closely regulated by a number of processes, but especially by neurovascular coupling [21]. The component processes are controlled through coordinated activity of neurons, astrocytes, and parenchymal arterioles. Changes in localized blood flow ensure adequate oxygenation and nutrition to brain tissues. The cascade that begins and continues this process is complex, involving glutamate receptors on astrocytes, and potassium efflux mediated by BK channels on astrocyte end feet [21]. Modulation/activation of BK channels appears to be a prime mechanism in the transition from vasodilation in normal oxygenation of brain tissue, to vasoconstriction in the presence of blood as a consequence of a cerebral aneurysm rupture, worsening cerebral ischemia, and damage to cells [21].

BK channel subtypes may also play a role during cerebral ischemia from stroke other than aneurysmal bleed. Focal ischemia occurs due to the activity of BK channels on astrocytes increasing intracellular calcium and potassium efflux resulting in apoptotic and necrotic cellular death and reactive gliosis, all of which extend the damage from the initial ischemic event [22].

In a 2019 study using a rat model, it was found that BK channels are a target for vitamin C [23]. Vitamin C intake correlates with a lower incidence of stroke (but not with lower incidence of myocardial infarction) [24]. There have been animal as well as human studies testing vitamin $\mathrm{C}$ for the prevention of cerebral infarcts. Unfortunately, they have yielded contradictory results [23]. Initially it was thought that the protective effect was due to the antioxidant action of vitamin However, long-term ingestion of vitamin $\mathrm{C}$ is a different situation than is its use as a rescue therapeutic in an acute cerebral ischemic scenario. Giving low level vitamin $C$ over time reduced the impact of cerebral infarct size in this model, and it was proposed that BK channels were at least partially contributory to this effect. Interestingly, in acute studies using the rat model, activation of the BK channel, caused by cerebral ischemia increased cerebral circulation through cerebral artery smooth muscles, resulting in a compensatory increase in blood flow [25].

In summary, it appears that targeted BK channel modulation could offer a potential adjunctive therapy in the setting of cerebral ischemia. Clearly, much more research in this area is needed.

\section{Cardioprotection}

BK channels are extensively distributed in cardiovascular smooth muscle and 
cardiac fibroblasts, where they play a role as mediators of inflammation and in the remodeling of the heart following ischemic injury [26] [27]. Multiple studies in animal models have suggested a role for BK channels in cardioprotection prior to, and after, reperfusion and ischemic injuries. For example, Bentzen et al. demonstrated that BK channels are involved in protection of the heart against ischemia-reperfusion of isolated perfused rat hearts subjected to $35 \mathrm{~min}$ of global ischemia followed by $120 \mathrm{~min}$ of reperfusion injury [28]. Similar beneficial results were found on ischemia-reperfusion infarct size and lactate dehydrogenase release [29]. Additional studies contribute to this conclusion [30] [31] [32] [33].

Borchet et al. demonstrated that BK channels likely participate in the improved resistance demonstrated by chronically hypoxic rats against injury caused by metabolic inhibition induced by sodium cyanide, followed by 30-min reenergization [34]. Soltysinska et al. showed, using an ex-vivo model of ischemia-reperfusion injury, that the area of infarction in BK channel knockout $\left(\mathrm{BK}^{--}\right)$mice devoid of $\mathrm{BK}$ channels was approximately double that in wild-type mice (which express normal levels of BK channels) [35].

In conclusion, the role of $\mathrm{BK}$ channels in cardioprotection is well recognized, at least in animal models. Further research is needed to focus on the best option in human populations.

\section{Respiratory Stimulation}

Hypoxia-an abnormally low level of oxygen pressure $\left(\mathrm{pO}_{2}\right)$ in arterial blood-is a principal physiological alerting signal for maintaining normal oxygen homeostasis. The body's response to hypoxia is reflexive and rapid (in the absence of respiratory-depressant influences), with the primary sensing organs (containing chemoceptors) being the carotid bodies at the bifurcation of the carotid arteries. Although similar sensors are found at the aortic arch and in the abdominal arteries, it is the carotid bodies that most respond to hypoxia [36]. The carotid bodies also respond to increase in carbon dioxide $\left(\mathrm{pCO}_{2}\right)$ and decrease in $\mathrm{pH}$. Under basal and normal conditions $\left(\mathrm{pO}_{2}-100 \mathrm{mmHg}\right)$, sensory carotid body signaling is low. But signaling increases dramatically with even a small decrease in arterial blood $\mathrm{pO}_{2}$, occurring within seconds. Hypoxia-sensitivity can differ between populations (e.g., strains of mice), but is maintained within very close limits within an individual [37]. Human twin studies suggest a genetically-inherited determinant of sensitivity to hypoxia [38].

The response of the carotid body to changes in blood gases and $\mathrm{pH}$ is noteworthy for its sensitivity, speed, and lack of adaptation over time [39]. One of the most powerful ligands and best characterized effectors of the BK channel is carbon monoxide (CO) [40]. CO activates BK channels via both direct and indirect mechanisms [40]. Although it was originally thought that hypoxia directly depolarizes the glomus cells of the carotid body, thereby inhibiting potassium outward currents, recent evidence points to the BK channels [41]. The data suggest that the BK channels are activated in the presence of hypoxia, as a sort of 
negative-feedback loop [27]. Once the carotid body is stimulated by hypoxia, a variety of strong ventilatory autonomic, cardiovascular, renal, and endocrine responses are elicited [42]. Stimulation of BK channels in the carotid body induces neurotransmitter release and increased number of action potentials in the glossopharyngeal nerve [43]. The impulse excites the nucleus of the solitary tract which targets brainstem circuits and stimulates respiratory response elements. The increase in respiratory response results in an increase in tidal volume (volume of air displaced between inhalation and exhalation) as well as the respiratory rate. Due to normal cardiopulmonary coupling, there is a concomitant increase in cardiac output [43]. The hypoxic drive is strong enough to stimulate breathing even during hypocapnic apneas, such as occurs during opioid-induced respiratory depression and opioid overdose [43].

In conclusion, BK channels offer a clear target in the setting of hypoxia- and hypercapnia-reduced respiratory function. Further, BK channels have also been shown to be involved in the regulation of airway surface liquid (ASL) homeostasis, and therefore mucociliary clearance (MCC), both important innate host defense mechanisms [44]. In disease states where ASL volume is reduced and pathology ensues, targeting BK channels may be a viable pharmacologic target.

\section{Discussion}

The accumulated evidence suggests that BK channels-because of the extensive diversity of their subtypes (subunit composition, 3-D arrangement, and accessory molecules), distribution, and pharmacology-participate in the maintenance of protection against ischemia and hypoxia at multiple points throughout the body. They appear to be major players in providing cerebro- and cardio-protective actions against ischemia and hypoxia, as well as stimulate respiration in the presence of such conditions (both local and systemic). Although individual examples of organ protection have been widely published, we propose that BK channels participate in a coordinated control system. That is, that they work in concert to provide protection against ischemia and hypoxia not only where it occurs, but also preventing negative consequences at distal sites.

We cite as an example, stimulation of respiration. In the wake of the recent COVID pandemic, opioid- and polysubstance overdose deaths are nearing an all-time high. Deaths have dramatically increased due to high-potency illicit fentanoids and to polysubstance abuse [45]. The only respiratory-stimulant drug currently approved in the United States is doxapram. Its mechanism of action appears to mainly involve inhibition of TASK (TWIK (Tandem of P-domains in a Weakly Inward rectifying $\mathrm{K}^{+}$channel)-related Acid Sensitive $\mathrm{K}^{+}$) channels [46]. ENA-001, currently in clinical trials, appears to work mainly as a regulator at BK channels in the carotid bodies [47]. It has received attention as an "agnostic" respiratory stimulant, since it can counteract the effects of a number of diverse respiratory depressants (opioid, benzodiazepine, isoflurane, and Propofol) [48].

Some other physiological processes in which BK channel (subtypes) are 
thought to play an important role include smooth muscle contraction, hormone secretion, neural excitation, and circadian rhythms [49]. The common link appears to be that BK channel action maintains a balance between over- and under-stimulation, helping shape normal activity and maintain homeostasis [15]. Therefore, numerous disorders may well be linked to BK channel dysfunction. For example, it has been suggested that BK channels in vascular smooth muscle could be targeted for treating diabetic complications and bladder dysfunctions [50] [51]. The BK channel activator unoprostone is approved for the treatment of ocular hypertension [52] [53]. It is also speculated that modulation of these channels could potentially play a role in the treatment of schizophrenia, autism, and other psychiatric disorders [54]. It will be interesting to see whether improvement of these seemingly disparate problems could be obtained using novel BK channel subtype directed drugs.

\section{Conclusion}

There is emerging and accumulating evidence that the "BK" subfamily of large-conductance potassium channels is involved in a diverse variety of physiological processes, and perhaps coordinated, protective or counteractive responses to local or generalized ischemia and hypoxia. They are widely distributed, yet physiological differences among BK channels bestow localized differences in subunit composition, distribution, $2^{\text {nd }}$-messenger coupling, and pharmacologic properties. BK channels have many potential therapeutic applications in the maintenance of oxygen homeostasis, cerebro- and cardio-protection, and stimulation of respiration in response to drug-induced respiratory depression. BK channels may also offer other promising targets for novel pharmaceutical development.

\section{Conflicts of Interest}

The authors declare no conflicts of interest regarding the publication of this paper.

\section{Disclosures}

Alexander Kraus is a scientific advisor to Enalare Therapeutics and (within the last 12 months) declares consultation for Spirify Pharmaceuticals, Develco Pharma Schweiz, Celanese, Cellis, Onena Medicines, MaK Medicine, and Princeton Capital Advisors. Robert B. Raffa is CSO of Neumentum, Co-founder of Enalare and CaRafe, and within the previous 12 months was consultant for Bridge Therapeutics and BDSI. John F. Peppin (within the last 12 months) declares consultation for Relmada Therapeutics and Enalare therapeutics. Joseph V. Pergolizzi Jr. discloses the following relationships: Consultant/ Speaker, Owner, and Researcher for Spirify, US World Meds, Salix, Enalare, Scilex, Pfizer, Lilly, Teva, Taketa, Regeneron, Grunenthal, Neumentum, BDSI and Bridge Therapeutics. 


\section{References}

[1] Santos, R., Ursu, O., Gaulton, A., et al. (2017) A Comprehensive Map of Molecular Drug Targets. Nature Reviews Drug Discovery, 16, 19-34. https://doi.org/10.1038/nrd.2016.230

[2] Sriram, K. and Insel, P.A. (2018) G Protein-Coupled Receptors as Targets for Approved Drugs: How Many Targets and How Many Drugs? Molecular Pharmacology, 93, 251-258. https://doi.org/10.1124/mol.117.111062

[3] Kshatri, A.S., Gonzalez-Hernandez, A. and Giraldez, T. (2018) Physiological Roles and Therapeutic Potential of $\mathrm{Ca}^{2+}$ Activated Potassium Channels in the Nervous System. Frontiers in Molecular Neuroscience, 11, 258. https://doi.org/10.3389/fnmol.2018.00258

[4] Yang, H., Zhang, G. and Cui, J. (2015) BK Channels: Multiple Sensors, One Activation Gate. Frontiers in Physiology, 6, 29. https://doi.org/10.3389/fphys.2015.00029

[5] Adelman, J.P., Shen, K.Z., Kavanaugh, M.P., et al. (1992) Calcium-Activated Potassium Channels Expressed from Cloned Complementary DNAs. Neuron, 9, 209-216. https://doi.org/10.1016/0896-6273(92)90160-F

[6] Pantazis, A. and Olcese, R. (2016) Biophysics of BK Channel Gating. International Review of Neurobiology, 128, 1-49. https://doi.org/10.1016/bs.irn.2016.03.013

[7] Lee, U.S. and Cui, J. (2010) BK Channel Activation: Structural and Functional Insights. Trends in Neurosciences, 33, 415-423. https://doi.org/10.1016/j.tins.2010.06.004

[8] Zhang, G., Huang, S.Y., Yang, J., et al. (2010) Ion Sensing in the RCK1 Domain of BK Channels. Proceedings of the National Academy of Sciences of the United States of America, 107, 18700-18705. https://doi.org/10.1073/pnas.1010124107

[9] Esser, M., Guy Jr., G., Zhang, K. and Brewer, R. (2019) Binge Drinking and Prescription Opioid Misuse in the US, 2012-2014. American Journal of Preventive Medicine, 57, 197-208. https://doi.org/10.1016/j.amepre.2019.02.025

[10] Chen, L., Jeffries, O., Rowe, I.C.M., et al. (2010) Membrane Trafficking of Large Conductance Calcium-Activated Potassium Channels Is Regulated by Alternative Splicing of a Transplantable, Acidic Trafficking Motif in the RCK1-RCK2 Linker. Journal of Biological Chemistry, 285, 23265-23275. https://doi.org/10.1074/jbc.M110.139758

[11] Wu, Y., Yang, Y., Ye, S. and Jiang, Y. (2010) Structure of the Gating Ring from the Human Large-Conductance $\mathrm{Ca}^{2+}$-Gated $\mathrm{K}^{+}$Channel. Nature, 466, 393-397. https://doi.org/10.1038/nature09252

[12] Ishii, T.M., Maylie, J. and Adelman, J.P. (1997) Determinants of Apamin and d-Tubocurarine Block in SK Potassium Channels. Journal of Biological Chemistry, 272, 23195-23200. https://doi.org/10.1074/jbc.272.37.23195

[13] Griguoli, M., Sgritta, M. and Cherubini, E. (2016) Presynaptic BK Channels Control Transmitter Release: Physiological Relevance and Potential Therapeutic Implications. The Journal of Physiology, 594, 3489-3500. https://doi.org/10.1113/JP271841

[14] Gonzalez-Perez, V. and Lingle, C.J. (2019) Regulation of BK Channels by Beta and Gamma Subunits. Annual Review of Physiology, 81, 113-137.

https://doi.org/10.1146/annurev-physiol-022516-034038

[15] Contet, C., Goulding, S.P., Kuljis, D.A. and Barth, A.L. (2016) BK Channels in the Central Nervous System. International Review of Neurobiology, 128, 281-342. https://doi.org/10.1016/bs.irn.2016.04.001

[16] Ramírez-Latorre, J.A. (2012) Functional Upregulation of $\mathrm{Ca}^{2+}$-Activated $\mathrm{K}^{+}$Chan- 
nels in the Development of Substantia Nigra Dopamine Neurons. PLoS ONE, 7, e51610. https://doi.org/10.1371/journal.pone.0051610

[17] Selemon, L.D. (2013) A Role for Synaptic Plasticity in the Adolescent Development of Executive Function. Translational Psychiatry, 3, e238.

https://doi.org/10.1038/tp.2013.7

[18] Lo, E.H., Dalkara, T. and Moskowitz, M.A. (2003) Mechanisms, Challenges and Opportunities in Stroke. Nature Reviews Neuroscience, 4, 399-415. https://doi.org/10.1038/nrn1106

[19] Gribkoff, V.K., Starrett Jr., J.E., Dworetzky, S.I., et al. (2001) Targeting Acute Ischemic Stroke with a Calcium-Sensitive Opener of Maxi-K Potassium Channels. Nature Medicine, 7, 471-477. https://doi.org/10.1038/86546

[20] Jensen, B.S. (2002) BMS-204352: A Potassium Channel Opener Developed for the Treatment of Stroke. CNS Drug Reviews, 8, 353-360. https://doi.org/10.1111/j.1527-3458.2002.tb00233.x

[21] Koide, M., Bonev, A.D., Nelson, M.T. and Wellman, G.C. (2012) Inversion of Neurovascular Coupling by Subarachnoid Blood Depends on Large-Conductance $\mathrm{Ca}^{2+}$-Activated $\mathrm{K}^{+}(\mathrm{BK})$ Channels. Proceedings of the National Academy of Sciences of the United States of America, 109, E1387-1395.

https://doi.org/10.1073/pnas.1121359109

[22] Yi, M., Wei, T., Wang, Y., et al. (2017) The Potassium Channel KCa3.1 Constitutes a Pharmacological Target for Astrogliosis Associated with Ischemia Stroke. Journal of Neuroinflammation, 14, Article No. 203. https://doi.org/10.1186/s12974-017-0973-8

[23] Li, L., Li, S., Hu, C., et al. (2019) $\mathrm{BK}_{\mathrm{Ca}}$ Channel Is a Molecular Target of Vitamin C to Protect against Ischemic Brain Stroke. Molecular Membrane Biology, 35, 9-20. https://doi.org/10.1080/09687688.2019.1608378

[24] Del Rio, D., Agnoli, C., Pellegrini, N., et al. (2011) Total Antioxidant Capacity of the Diet Is Associated with Lower Risk of Ischemic Stroke in a Large Italian Cohort. The Journal of Nutrition, 141, 118-123. https://doi.org/10.3945/jn.110.125120

[25] Rottgen, T., Dick, G., Chantler, P. and Frisbee, J. (2016) Increased Function of Large-Conductance of Potassium Channels following Acute Ischemic Stroke. The FASEB Journal, 30, 1224.

[26] Szteyn, K. and Singh, H. (2020) $\mathrm{BK}_{\mathrm{Ca}}$ Channels as Targets for Cardioprotection. Antioxidants (Basel), 9, 760. https://doi.org/10.3390/antiox9080760

[27] Wang, Y.J., Sung, R.J., Lin, M.W. and Wu, S.N. (2006) Contribution of $\mathrm{BK}_{\mathrm{Ca}}$-Channel Activity in Human Cardiac Fibroblasts to Electrical Coupling of Cardiomyocytes-Fibroblasts. The Journal of Membrane Biology, 213, 175-185. https://doi.org/10.1007/s00232-007-0027-8

[28] Bentzen, B.H., Osadchii, O., Jespersen, T., et al. (2009) Activation of Big Conductance $\mathrm{Ca}^{2+}$-Activated $\mathrm{K}^{+}$Channels (BK) Protects the Heart against Ischemia-Reperfusion Injury. Pflügers Archiv, 457, 979-988. https://doi.org/10.1007/s00424-008-0583-5

[29] Cao, C.M., Xia, Q., Gao, Q., Chen, M. and Wong, T.M. (2005) Calcium-Activated Potassium Channel Triggers Cardioprotection of Ischemic Preconditioning. Journal of Pharmacology and Experimental Therapeutics, 312, 644-650. https://doi.org/10.1124/jpet.104.074476

[30] Ohya, S., Kuwata, Y., Sakamoto, K., Muraki, K. and Imaizumi, Y. (2005) Cardioprotective Effects of Estradiol Include the Activation of Large-Conductance $\mathrm{Ca}^{2+}$-Activated $\mathrm{K}^{+}$Channels in Cardiac Mitochondria. American Journal of Physi- 
ology-Heart and Circulatory Physiology, 289, H1635-H1642.

https://doi.org/10.1152/ajpheart.00016.2005

[31] Sato, T., Saito, T., Saegusa, N. and Nakaya, H. (2005) Mitochondrial Ca ${ }^{2+}$-Activated $\mathrm{K}^{+}$Channels in Cardiac Myocytes: A Mechanism of the Cardioprotective Effect and Modulation by Protein Kinase A. Circulation, 111, 198-203.

https://doi.org/10.1161/01.CIR.0000151099.15706.B1

[32] Wang, X., Fisher, P.W., Xi, L. and Kukreja, R.C. (2008) Essential Role of Mitochondrial $\mathrm{Ca}^{2+}$-Activated and ATP-Sensitive $\mathrm{K}^{+}$Channels in Sildenafil-Induced Late Cardioprotection. Journal of Molecular and Cellular Cardiology, 44, 105-113. https://doi.org/10.1016/j.yjmcc.2007.10.006

[33] Xu, W., Liu, Y., Wang, S., et al. (2002) Cytoprotective Role of $\mathrm{Ca}^{2+}$-Activated $\mathrm{K}^{+}$ Channels in the Cardiac Inner Mitochondrial Membrane. Science, 298, 1029-1033. https://doi.org/10.1126/science.1074360

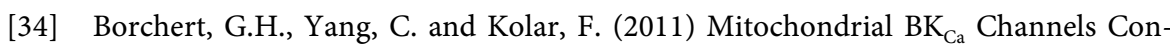
tribute to Protection of Cardiomyocytes Isolated from Chronically Hypoxic Rats. American Journal of Physiology-Heart and Circulatory Physiology, 300, H507-H513. https://doi.org/10.1152/ajpheart.00594.2010

[35] Soltysinska, E., Bentzen, B.H., Barthmes, M., et al. (2014) KCNMA1 Encoded Cardiac BK Channels Afford Protection against Ischemia-Reperfusion Injury. PLoS ONE, 9, e103402. https://doi.org/10.1371/journal.pone.0103402

[36] Kumar, P. and Prabhakar, N.R. (2012) Peripheral Chemoreceptors: Function and Plasticity of the Carotid Body. Comprehensive Physiology, 2, 141-219.

https://doi.org/10.1002/cphy.c100069

[37] Otsubo, T., Kostuk, E.W., Balbir, A., Fujii, K. and Shirahata, M. (2011) Differential Expression of Large-Conductance $\mathrm{Ca}^{2+}$-Activated $\mathrm{K}$ Channels in the Carotid Body between DBA/2J and A/J Strains of Mice. Frontiers in Cellular Neuroscience, 5, 19. https://doi.org/10.3389/fncel.2011.00019

[38] Thomas, D.A., Swaminathan, S., Beardsmore, C.S., et al. (1993) Comparison of Peripheral Chemoreceptor Responses in Monozygotic and Dizygotic Twin Infants. American Review of Respiratory Disease, 148, 1605-1609. https://doi.org/10.1164/ajrccm/148.6_Pt_1.1605

[39] Prabhakar, N.R., Peng, Y.J. and Nanduri, J. (2018) Recent Advances in Understanding the Physiology of Hypoxic Sensing by the Carotid Body. F1000Research, 7, F1000 Faculty Rev-1900. https://doi.org/10.12688/f1000research.16247.1

[40] Kim, H.H. and Choi, S. (2018) Therapeutic Aspects of Carbon Monoxide in Cardiovascular Disease. International Journal of Molecular Sciences, 19, 2381. https://doi.org/10.3390/ijms19082381

[41] Wang, J. and Kim, D. (2018) Activation of Voltage-Dependent $\mathrm{K}^{+}$Channels Strongly Limits Hypoxia-Induced Elevation of $\left[\mathrm{Ca}^{2+}\right]_{\mathrm{i}}$ in Rat Carotid Body Glomus Cells. The Journal of Physiology, 596, 3119-3136. https://doi.org/10.1113/JP275275

[42] Iturriaga, R. (2018) Translating Carotid Body Function into Clinical Medicine. The Journal of Physiology, 596, 3067-3077. https://doi.org/10.1113/JP275335

[43] Lindsey, B.G., Nuding, S.C., Segers, L.S. and Morris, K.F. (2018) Carotid Bodies and the Integrated Cardiorespiratory Response to Hypoxia. Physiology (Bethesda), 33, 281-297. https://doi.org/10.1152/physiol.00014.2018

[44] Kis, A., Krick, S., Baumlin, N. and Salathe, M. (2016) Airway Hydration, Apical K ${ }^{+}$ Secretion, and the Large-Conductance, $\mathrm{Ca}^{2+}$-Activated and Voltage-Dependent Potassium (BK) Channel. Annals of the American Thoracic Society, 13, S163-S168.

[45] Peppin, J.F., Raffa, R.B. and Schatman, M.E. (2020) The Polysubstance Over- 
dose-Death Crisis. Journal of Pain Research, 13, 3405-3408. https://doi.org/10.2147/JPR.S295715

[46] Couch, G., White, M. and de Gray, L. (2020) Central Nervous System Stimulants: Basic Pharmacology and Relevance to Anaesthesia and Critical Care. Anaesthesia \& Intensive Care Medicine, 21, 503-511. https://doi.org/10.1016/j.mpaic.2020.07.005

[47] McCartney, C.E., McClafferty, H., Huibant, J.-M., et al. (2005) A Cysteine-Rich Motif Confers Hypoxia Sensitivity to Mammalian Large Conductance Voltage- and Ca-Activated K (BK) Channel Alpha-Subunits. Proceedings of the National Academy of Sciences of the United States of America, 102, 17870-17876. https://doi.org/10.1073/pnas.0505270102

[48] McLeod, J.F., Leempoels, J.M., Peng, S.X., et al. (2014) GAL-021, a New Intravenous $\mathrm{BK}_{\mathrm{Ca}}$-Channel Blocker, Is Well Tolerated and Stimulates Ventilation in Healthy Volunteers. British Journal of Anaesthesia, 113, 875-883. https://doi.org/10.1093/bja/aeu182

[49] Cohen, M.V., Yang, X. and Downey, J.M. (2010) $A_{2 b}$ Adenosine Receptors Can Change Their Spots. British Journal of Pharmacology, 159, 1595-1597. https://doi.org/10.1111/j.1476-5381.2010.00668.x

[50] Qian, L., Liu, X. and Wang, R. (2014) Role of $\mathrm{BK}_{\mathrm{Ca}}$ Channels in Diabetic Vascular Complications. Chinese Medical Journal, 127, 1775-1781.

[51] Parajuli, S.P., Zheng, Y.M., Levin, R. and Wang, Y.X. (2016) Big-Conductance $\mathrm{Ca}^{2+}$-Activated $\mathrm{K}^{+}$Channels in Physiological and Pathophysiological Urinary Bladder Smooth Muscle cells. Channels (Austin), 10, 355-364.

https://doi.org/10.1080/19336950.2016.1180488

[52] Harms, N.V. and Toris, C.B. (2013) Current Status of Unoprostone for the Management of Glaucoma and the Future of Its Use in the Treatment of Retinal Disease. Expert Opinion on Pharmacotherapy, 14, 105-113. https://doi.org/10.1517/14656566.2013.748038

[53] Colwell, C.S. (2006) BK Channels and Circadian Output. Nature Neuroscience, 9, 985-986. https://doi.org/10.1038/nn0806-985

[54] Zaman, T., De Oliveira, C., Smoka, M., et al. (2017) BK Channels Mediate Synaptic Plasticity Underlying Habituation in Rats. Journal of Neuroscience, 37, 4540-4551. https://doi.org/10.1523/JNEUROSCI.3699-16.2017 